\title{
Radiation-induced glioblastoma with rhabdoid characteristics following treatment for medulloblastoma: A case report and review of the literature
}

\author{
YING WANG $^{1 *}$, SHUJUN SONG $^{2 *}$, XIAOMING $\mathrm{SU}^{1}$, JIHUA WU $^{3}, \mathrm{ZHUOJIE} \mathrm{DAI}^{1}$, DI CUI ${ }^{1}$, \\ YE RENG $^{1}$, JINGJING FAN ${ }^{1}$, YULONG SHEN ${ }^{1}$, QINGQIN WU ${ }^{1}$ and ZONGYE WANG ${ }^{1}$ \\ ${ }^{1}$ Department of Radiotherapy, ${ }^{2}$ Center for Special Medicine and Experimental Research, and \\ ${ }^{3}$ Department of Pathology, 306th Hospital of PLA, Beijing 100101, P.R. China
}

Received April 9, 2018; Accepted June 11, 2018

DOI: $10.3892 / \mathrm{mco} .2018 .1703$

\begin{abstract}
Glioblastoma multiforme (GBM) is a high-grade glioma that may develop from several other central nervous system tumors after radiation therapy. We herein report a case of GBM occurring 8 years after radiation therapy for medulloblastoma. The secondary tumor was histologically distinctly different from the primary tumor. Previously reported cases indicate that GBM induced by radiation therapy is associated with a highly aggressive clinical course with a high risk of early recurrence and poor prognosis. In addition, histological examination revealed that the tumor cells exhibited characteristics of both GBM and rhabdoid tumor cells. The diverse pathological characteristics of GBM may reflect the potential effects of radiation therapy on the tumor.
\end{abstract}

\section{Introduction}

As the survival of patients with brain tumors has increased with the advances in radiotherapy or intensive chemotherapy, the risk of secondary brain tumors has also increased significantly. Glioblastoma multiforme (GBM) is a high-grade glioma that may develop from several other tumors, such as medulloblastoma (MB), germinomas and Burkitt's lymphoma, after radiation therapy (1). The first case of radiation-induced GBM developing from MB following radiotherapy of the central nervous system (CNS) was reported by Kleriga et al in 1978 (2). Several similar cases have since been reported. The majority of radiation-induced glioblastomas (RIGs) may develop after a long latency period, and appear more frequently in the same location as the irradiation field. Furthermore,

Correspondence to: Dr Zongye Wang, Department of Radiotherapy, 306th Hospital of PLA, 9 North Anxiang Road, Beijing 100101, P.R. China

E-mail: wangzye@163.com

Key words: medulloblastoma, glioblastoma, radiotherapy-induced tumors, rhabdoid previous studies on RIGs report a more aggressive course and poorer prognosis compared with typical GBM.

Glioblastoma with rhabdoid characteristics was first described by Wyatt-Ashmead in 2001 (3) as an extremely rare occurrence. An increasing number of previous reports indicated that glioblastoma with rhabdoid components exhibited a highly aggressive course and often tended to occur in younger patients compared with typical GBM.

We herein report a case of RIG with rhabdoid characteristics in a 13-year-old boy who had received CNS radiotherapy for MB of the posterior fossa 8 years earlier.

\section{Case report}

A 4-year-old boy was first admitted to the hospital in April 2009 with headache, vomiting and gait disturbance. None of his family members had any known genetic diseases predisposing to cancer. Magnetic resonance imaging (MRI) of brain revealed an enhanced space-occupying lesion in the fourth ventricle. This lesion was completely resected, and was diagnosed as MB in April 2009 by postoperative histological examination. Postoperative radiotherapy was performed, with a dose of 30.6 Gy delivered to the craniospinal region and an additional delivery of $54 \mathrm{~Gy}$ to the posterior fossa after craniospinal irradiation. Follow-up MRI or CT scans were regularly performed thereafter, and revealed no evidence of recurrence over the next 8 years.

On January 2017, the patient was readmitted with weakness of the distal left limbs without objective loss of sensation, gait disturbance and ipsilateral angular salivation. Subsequently, a brain MRI revealed a quasi-circular cystic lesion with a significantly enhanced capsule in the right frontal lobe. The lesion was poorly circumscribed, without obvious peritumoral edema. The cystic component displayed inhomogeneous low signal on T1-weighted images, and inhomogeneous hyperintense signal on T2-weighted images. On February 2017, the patient underwent surgical resection, and the subsequent histological diagnosis was GBM. Intraoperatively, the main component of the lesion was cystic, and the wall of the cystic lesion exhibited inhomogeneous thickness. After surgery, the weakness of the left limbs worsened and 2 months later the 
patient was readmitted with paroxysmal headache. A brain MRI revealed multiple enhanced tissues adjacent to the tumor bed in the right frontal lobe (Fig. 1). Subsequently, the patient received 4 cycles of chemotherapy with nimotuzumab, bevacizumab and irinotecan (dosage records unavailable), following which there remained no evidence, from regular reexaminations, to indicate tumor progression.

The first diagnosed tumor displayed characteristics typical of MB: Pleomorphic tumor cells with sparse cytoplasm, hyperchromatic nuclei, extensive presence of apoptosis/necrosis and mitotic figures, with Homer-Wright rosettes (Fig. 2A). Immunohistochemical examination revealed expression of synaptophysin (Syn) in the cytoplasm of some MB cells (Fig. 2B). However, neurofilament protein, S-100 and vimentin (Vim) were not expressed in the tumor tissue.

Histological examination of the secondary tumor revealed that the neoplastic cells exhibited characteristics of both glioblastoma and rhabdoid tumor cells. The majority of the tumor cells exhibited microvascular proliferation, obvious atypia, abundant cytoplasm, extensive apoptosis and mitotic figures, as well as patchy necrosis. A few cells were characterized by eccentric nuclei with prominent nucleoli. Furthermore, scattered giant cells were identified. Immunohistochemically, the tumor cells were strongly positive for glial fibrillary acidic protein (GFAP), Vim and Olig-2. In addition, S-100, p53, isocitrate dehydrogenase 1 and $\alpha$-thalassemia/mental retardation syndrome $\mathrm{X}$-linked (ATRX) were also expressed by these tumor cells, and smooth muscle actin (SMA) was focally positive, reflecting the loss of integrase interactor-1 (INI-1) protein. However, there were no observations indicating primitive neuroectodermal tumor in this case. The molecular pathology revealed ATRX mutation and $\mathrm{O}^{6}$-methylguanine-DNA-methyltransferase promoter methylation. Examination by fluorescence in situ hybridization demonstrated that there was no loss of heterozygosity on chromosome 1p19q. The Ki-67 index (Ki 67-positive cells) was $30-40 \%$ (Fig. 3). Unfortunately, the patient died 1 year following the secondary recurrence.

\section{Discussion}

Glioblastoma is a high-grade glioma that may develop as a secondary tumor following radiation therapy. As reported in the first animal experiment conducted by Haymaker et al in 1972 (4), glioblastoma was induced by radiation in a monkey. Over the last 45 years, 296 cases of radiation-induced brain tumors, such as meningiomas, sarcomas and gliomas, have been reported. The criteria of radiation-induced glioblastoma established by Cahan et al (5) were as follows: i) The phenotypes of the primary and secondary tumors were distinctly different; ii) the site of the secondary tumor was within the field of irradiation; iii) the secondary tumor should be histologically diagnosed; iv) there should be a prolonged latency period between the treatment of the primary and the appearance of the secondary tumor. The case in our report was diagnosed as glioblastoma by histological examination 8 years after radiotherapy for MB.

In a study by Minniti et al (6), the risk of secondary brain tumor following conservative surgery and radiotherapy for pituitary adenoma was $2.4 \%$ in 20 years. Similar to these results, scientists in Margaret Hospital reported that the estimated cumulative risk for RIG was $1.7-2.7 \%$ at 15 years after
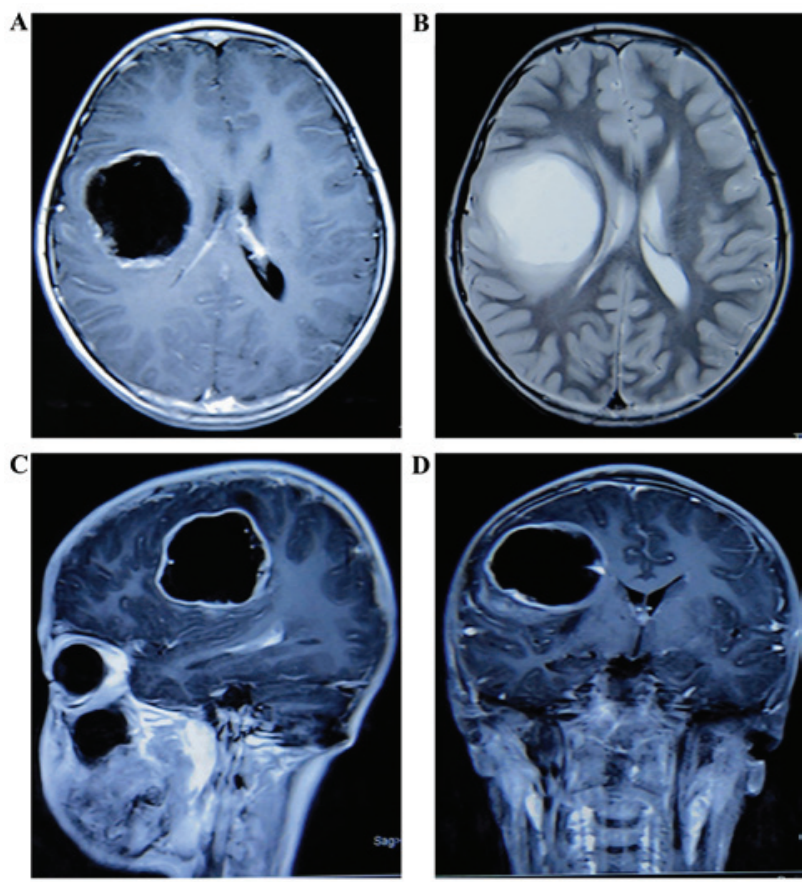

Figure 1. Magnetic resonance imaging of the brain in 2017. (A) Axial T1-weighted post-gadolinium image showing an inhomogeneous low-signal quasi-circular cystic lesion in the right frontal lobe. (B) Axial T2-weighted image showing inhomogeneous hyperintense signal of the cystic component. (C) Sagittal plane. (D) Coronary plane.
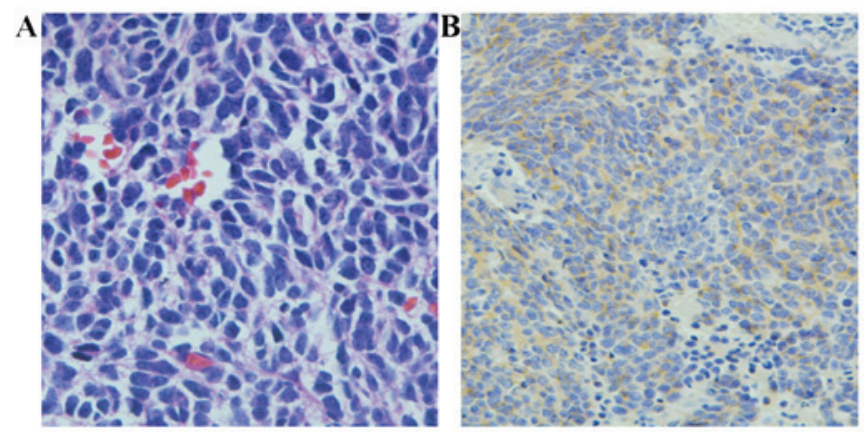

Figure 2. Microscopic characteristics of medulloblastoma. (A) The original tumor was composed of tumor cells with sparse cytoplasm, hyperchromatic nuclei, extensive apoptosis/necrosis and mitotic figures, with typical characteristics of medulloblastomas (hematoxylin and eosin staining; magnification, $x 400$ ). (B) Immunohistochemical staining showing that the tumor cells were strongly positive for synaptophysin (magnification, x200).

radiation therapy (7). RIG may develop from primary tumors such as hematological malignancies, MB, pituitary adenoma, low-grade glioma and craniopharyngioma. As demonstrated by previous data, MB accounts for $12.8 \%$ of primary tumors (8).

The majority of radiation-induced brain tumors (RIBTs) are reported in children who were aged $<10$ years when they initially received radiotherapy. The first case of RBIT reported by Kleriga et al (2), was a patient treated for MB at the age of 10 months and developed a malignant astrocytoma in the same location 11 years after radiotherapy. You et al reported 5 cases of secondary brain tumors; all these cases were irradiated at an age of $<10$ years (1). The age of patients who develop RIGs is frequently lower compared with that of spontaneous high-grade gliomas. The case of RIG reported herein had a history of 

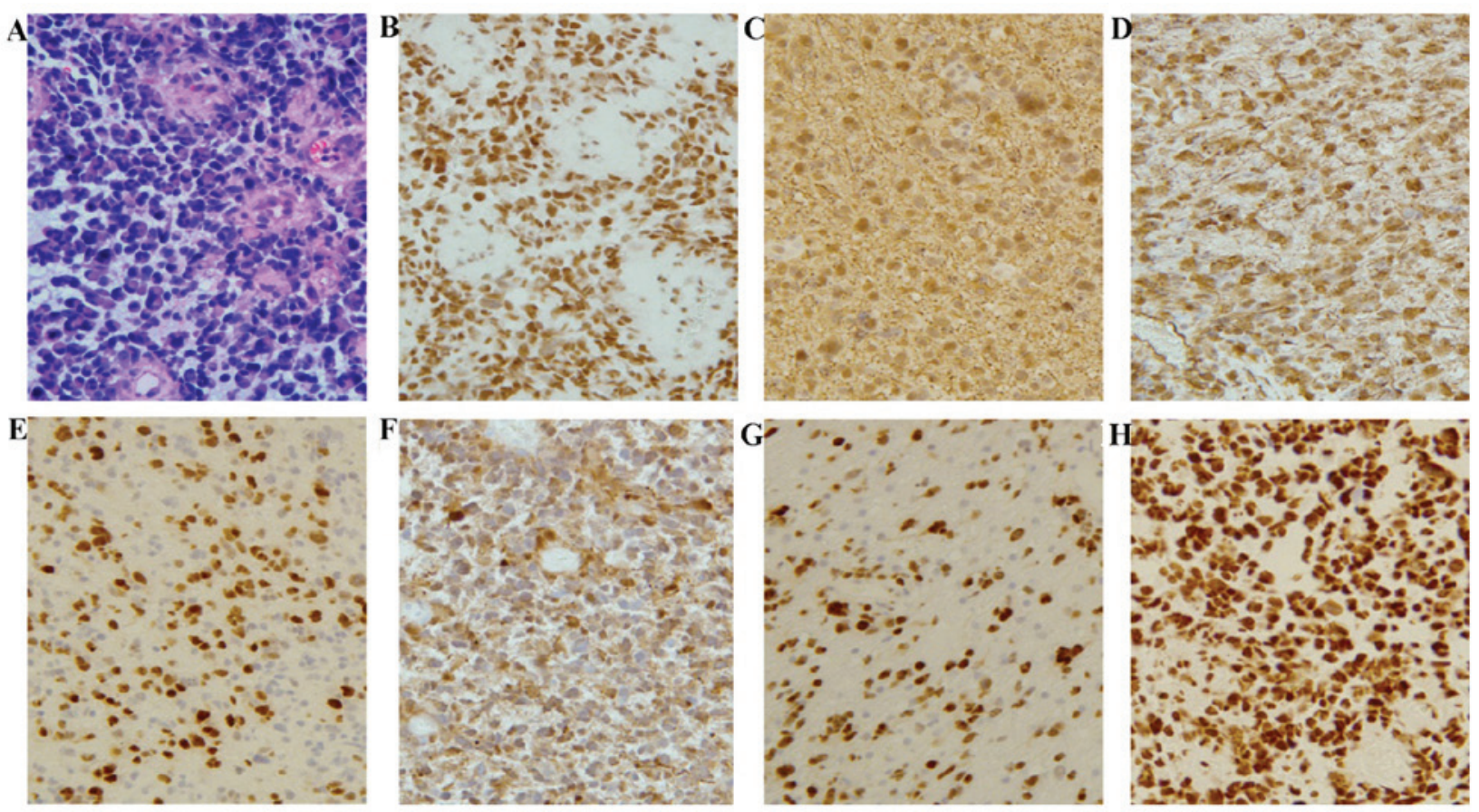

Figure 3. Histological and immunohistochemical characteristics of glioblastoma multiforme. (A) The histological characteristics of the secondary tumor were obviously different from those of the original tumor. Glioblastoma cells exhibited microvascular proliferation, obvious atypia, abundant cytoplasm, extensive apoptosis/necrosis and mitotic figures, as well as patchy necrosis. A few cells were characterized by eccentrically located nuclei with prominent nucleoli (hematoxylin and eosin staining; magnification, x200). (B) The tumor cell bearing characteristics of rhabdoid cells exhibited loss of the INI-1 protein compared with the surrounding INI-1-expressing cells (magnification, x200). (C-H) Immunohistochemically, the tumor cells highly expressed (C) GFAP, (D) vimentin and (E) Olig-2. (F) S-100, (G) p53, and (H) ATRX were also expressed in those tumor cells (magnification, x200). INI-1, integrase interactor 1; GFAP, glial fibrillary acidic protein; ATRX, $\alpha$-thalassemia/mental retardation syndrome X-linked.

radiotherapy for MB at the age of 4 years. These findings indicate that patient age at first radiotherapy may be an important factor in the development of RIBTs. Another factor affecting the development of RIGs is irradiation volume. The incidence of RIGs increased with increasing irradiation volume. According to a review of patients with secondary brain tumors induced by radiation therapy, the proportion of radiation-induced malignant gliomas was $75 \%$ among patients receiving initial radiotherapy of craniospinal or whole-brain fields (7). Our patient was treated with craniospinal irradiation at first admission and the secondary tumor occurred 8 years later within the radiation field.

A study by Yang et al (9) reported that the secondary tumor exhibited strong immunoreactivity for both GFAP and p53. These histological characteristics were also described in a case reported by Donson et al (10). Although p53 mutations were not detected, strong immunoreactivity for p53 is frequently associated with gene mutations of p53. p53 gene mutations have been identified in radiation-induced tumors $(11,12)$; thus, they may play a relevant role in the development of RIGs. However, the association between p53 gene mutations and the occurrence of secondary tumors remains largely unexplored.

In the present case, in addition to the strong expression of GFAP and p53, Vim, Syn and epithelial membrane antigen (EMA) were also expressed. As previously reported, Vim, SMA, EMA and Syn were mostly expressed in the secondary epithelioid/rhabdoid glioblastoma $(13,14)$. Furthermore, focal loss of the INI-1 protein was observed in rhabdoid tumor cells, which were characterized by eccentric nuclei with prominent nucleoli. Focal loss of the INI-1 protein was also observed in cases reported by Sugimoto et al (15). Similar findings were also reported by a study on four cases of rhabdoid glioblastoma (16). Unfortunately, all these cases had a poor prognosis, with a median survival from the time of diagnosis of rhabdoid glioblastoma of only $\sim 4.9$ months $(16,17)$.

The present case represents a rare occurrence of RIG with the characteristics of rhabdoid glioblastoma following CNS radiotherapy. Due to the extremely poor prognosis, this type of tumor should be further investigated and more studies are required to determine its relevance to irradiation.

\section{Acknowledgements}

Not applicable.

\section{Funding}

This manuscript was supported by the National Keypoint Research and Invention Program (grant no. 2016YFC0105705).

\section{Availability of data and materials}

All data generated during this study are included in this published article.

\section{Authors' contributions}

YW, SJS, ZW and XS conceived and designed the study. ZW, YW and ZD drafted the manuscript. JW, DC, YR, JF, QW and YS critically revised the manuscript for content. All authors approved the manuscript for submission. 


\section{Ethics approval and consent to participate}

Not applicable.

\section{Patient consent for publication}

The patient's parents provided written informed consent for the publication of the case details and associated images.

\section{Competing interests}

The authors declare that they have no competing interests.

\section{References}

1. You SH, Lyu CJ, Kim DS and Suh CO: Second primary brain tumors following cranial irradiation for pediatric solid brain tumors. Child's Nerv Syst 29: 1865-1870, 2013.

2. Kleriga E, Sher JH, Nallainathan SK, Stein SC and Sacher M: Development of cerebellar malignant astrocytoma at site of a medulloblastoma treated 11 years earlier. Case report. J Neurosurg 49: 445-449, 1978.

3. Wyatt-Ashmead J, Kleinschmidt-DeMasters BK, Hill DA, Mierau GW, McGavran L, Thompson SJ and Foreman NK: Rhabdoid glioblastoma. Clin Neuropathol 20: 248-255, 2001.

4. Haymaker W, Rubinstein LJ and Miquel J: Brain tumors in irradiated monkeys. Acta neuropathologica 20: 267-277, 1972.

5. Cahan WG, Woodard HQ, Higinbotham NL, Stewart FW and Coley BL: Sarcoma arising in irradiated bone: Report of eleven cases. 1948. Cancer 82: 8-34, 1998.

6. Minniti G, Traish D, Ashley S, Gonsalves A and Brada M: Risk of second brain tumor after conservative surgery and radiotherapy for pituitary adenoma: Update after an additional 10 years. J Clin Endocrinol Metab 90: 800-804, 2005.

7. Paulino AC, Mai WY, Chintagumpala M, Taher A and Teh BS: Radiation-induced malignant gliomas: is there a role for reirradiation? Int J Radiat Oncol Biol Phys 71: 1381-1387, 2008.
8. Yamanaka R, Hayano A and Kanayama T: Radiation-induced gliomas: A comprehensive review and meta-analysis. Neurosurg Rev 5, 2016 (Epub ahead of print).

9. Yang SY, Wang KC, Cho BK, Kim YY, Lim SY, Park SH, Kim IH and Kim SK: Radiation-induced cerebellar glioblastoma at the site of a treated medulloblastoma: Case report. J Neurosurg 102 (4 Suppl): 417-422, 2005.

10. Donson AM, Erwin NS, Kleinschmidt-DeMasters BK, Madden JR, Addo-Yobo SO and Foreman NK: Unique molecular characteristics of radiation-induced glioblastoma. J Neuropathol Exp Neurol 66: 740-749, 2007.

11. Gessi M, Maderna E, Guzzetti S, Cefalo G, Massimino M, Solero CL, Finocchiaro G and Pollo B: Radiation-induced glioblastoma in a medulloblastoma patient: a case report with molecular features. Neuropathology 28: 633-639, 2008.

12. Brachman DG, Hallahan DE, Beckett MA, Yandell DW and Weichselbaum RR: p53 gene mutations and abnormal retinoblastoma protein in radiation-induced human sarcomas. Cancer Res 51: 6393-6396, 1991.

13. Nagai S, Kurimoto M, Ishizawa S, Hayashi N, Hamada H, Kamiyama $\mathrm{H}$ and Endo $\mathrm{S}$ : A rare astrocytic tumor with rhabdoid features. Brain Tumor Pathol 26: 19-24, 2009.

14. Mutou J, Hirose Y, Ikeda E, Yoshida K, Nakazato Y and Kawase T: Malignant brain tumor with rhabdoid features in an adult. Neurol Med Chir (Tokyo) 51: 449-454, 2011.

15. Sugimoto K, Ideguchi M, Kimura T, Kajiwara K, Imoto H, Sadahiro H, Ishii A, Kawano H, Ikeda E and Suzuki M: Epithelioid/rhabdoid glioblastoma: A highly aggressive subtype of glioblastoma. Brain Tumor Pathol 33: 137-146, 2016.

16. Babu R, Hatef J, McLendon RE, Cummings TJ, Sampson JH, Friedman AH and Adamson C: Clinicopathological characteristics and treatment of rhabdoid glioblastoma. J Neurosurg 119: 412-419, 2013.

17. Byeon SJ, Cho HJ, Baek HW, Park CK, Choi SH, Kim SH, Kim HK and Park SH: Rhabdoid glioblastoma is distinguishable from classical glioblastoma by cytogenetics and molecular genetics. Hum Pathol 45: 611-620, 2014. 